\title{
Abstracts of Theses Approved for the MSc Degree at the Health Sciences Centre, Kuwait University
}

\author{
A Study of Nociceptin (Orphanin \\ FQ)-Glutamatergic Interaction in \\ Spinal Nociceptive Transmission \\ N.T. Syed \\ Department of Pharmacology and Toxicology, \\ Faculty of Medicine, Kuwait University, Kuwait
}

Introduction and Objective: Nociceptin (orphanin FQ), an endogenous, nonopioid peptide, produces pronociceptive effects at the supraspinal level and antinociceptive actions in the spinal cord. $L$-Glutamate participates in spinal nociceptive transmission and selective antagonists at glutamate receptors raise nociceptive thresholds. We investigated the possibility that nociceptin's analgesic action is through modulation of spinal glutamatergic processes. Materials and Methods: Adult male Sprague-Dawley rats $(\mathrm{n}=250)$ were implanted with an intrathecal catheter. The antinociceptive effects of nociceptin (1-40 $\mu \mathrm{g}$ ), DL-AP5 (NMDA receptor antagonist, 1-30 $\mu \mathrm{g}$ ), NBQX (AMPA/KA receptor antagonist, 0.5-10 $\mu \mathrm{g}$ ) and AIDA (mGlu $\mathrm{m}_{1 \& 5}$ receptor antagonist, $0.01-20 \mu \mathrm{g}$ ) were established using standard tail immersion, paw pressure (for acute pain) and formalin tests (for tonic pain). Combined effects of nociceptin $(5,10,20 \mu \mathrm{g})$ were studied with AP5 $(2.5 \mu \mathrm{g})$, NBQX $(1 \mu \mathrm{g})$ or AIDA $(0.5 \mu \mathrm{g})$ in acute pain tests. In the formalin test, nociceptin $(5 \mu \mathrm{g})$ was combined with AP5 $(1 \mu \mathrm{g})$, NBQX $(2.5 \mu \mathrm{g})$ or AIDA $(10 \mu \mathrm{g})$. Motor coordination was also assessed. Results: Nociceptin and glutamate receptor antagonists produced dose-related antinociceptive effects against three noxious stimuli. Nociceptin and AP5 only acted additively and subadditively against mechanical and chemical-induced nociception, respectively. Higher doses of AP5 and NBQX were associated with motor disturbances. Conclusion: The spinal antinociceptive effects of nociceptin and glutamate receptor antagonists were confirmed. It is suggested that nociceptin's antinociceptive effects against mechanical and chemical stimulation are mediated through NMDA receptor-dependent transmission but not that dependent on AMPA or $\mathrm{mGlu}_{1 \& 5}$ receptors. Furthermore, nociceptin's effects on heat-induced nociception do not appear to be dependent on glutamate receptor-mediated transmission.

C.W.T. Pilcher (supervisor)

M.S. Bitar (co-supervisor)

\section{Direct Detection of Mycobacterium tuberculosis in Clinical Samples by Multiplex Polymerase Chain Reaction}

\section{F.Y. Azizieh}

Department of Microbiology, Faculty of Medicine, Kuwait University, Kuwait

Objective: To develop multiplex polymerase chain reactions (PCRs) for specific identification and differentiation of Mycobacterium tuberculosis from other mycobacteria and to test their overall sensitivity and specificity as compared to the microbiological methods, microscopy and culture. Materials and Methods: Three different genes present in multiple copies in the M. tuberculosis genome, i.e. insertion sequence 6110 , IS1081 and the antigen 85 complex gene, were targeted. The selected primers amplified 245- and 375-bp DNA fragments from IS6110, a 248-bp DNA fragment from IS1081 and a 162-bp DNA fragment from the antigen 85 complex gene. Results: The amplification of all the products was specific to $M$. tuberculosis except the 162-bp DNA fragment, which was amplified from all mycobacteria. When tested individually with DNA obtained from cultured $M$. tuberculosis, the minimum quantity of DNA required for amplification was $1 \mathrm{fg}$ ( 0.2 bacteria) for the 245- and

\begin{tabular}{ll}
\hline KARGER & ( 2003 S. Karger AG, Basel \\
1011-7571/03/0123-0200\$19.50/0 \\
$\begin{array}{l}\text { Fax +4161306 12 34 } \\
\begin{array}{l}\text { E-Mail karger@karger.ch } \\
\text { www.karger.com }\end{array}\end{array}$ & $\begin{array}{l}\text { Accessible online at: } \\
\text { www.karger.com/mpp }\end{array}$
\end{tabular}

Fax + 416130612 www.karger.com 
162-bp DNA fragments, $10 \mathrm{fg}$ ( 2 bacteria) for the $375 \mathrm{bp}$ DNA fragment and $100 \mathrm{fg}$ (20 bacteria) for the 248-bp fragment. Single tube reactions were performed for simultaneous amplification of 375-, 248- and 162-bp DNA fragments (three-band multiplex PCR). The minimum quantity of DNA required for simultaneous amplification of all three products was $1 \mathrm{pg}$ (200 bacteria). The detection limit in single tube reaction for 245- and 162-bp fragments (two-band multiplex) was $10 \mathrm{fg}$ ( 2 bacteria) and $100 \mathrm{fg}$ (20 bacteria), respectively. The three-band and two-band multiplex PCRs as well as the amplification of the 245-bp DNA fragment in conventional one-band PCR were evaluated for sensitivity and specificity. A total of 263 clinical samples (sputum, CSF, aspirates, urine, pus and tissue biopsies etc.) were tested, of which 112 samples were positive by smear and/or culture. Overall sensitivity and specificity of PCR as compared to combined microbiological methods were 94 and $100 \%$, respectively. PCR detected 79 of 79 smear-positive (100\%) and 26 of 33 (79\%) smear-negative samples. Conclusion: The multiplex PCRs were useful in differentiating between $M$. tuberculosis complex and other mycobacteria. The highly sensitive PCR assay for detection of $M$. tuberculosis showed species specificity and improved sensitivity as compared to microscopic detection of mycobacteria.

A.S. Mustafa (supervisor)

T.D. Chugh (co-supervisor)

Supported by the College of Graduate Studies and Research Administration grant MI080, Kuwait University.

\section{Identification of Mycobacterium tuberculosis Antigens and Epitopes Activating T Cells of Protective Phenotype}

\section{N.M. Madi}

Department of Microbiology, Faculty of Medicine, Kuwait University, Kuwait

Objective: To identify antigens of Mycobacterium tuberculosis recognized by $\mathrm{T}$ cells of protective phenotype and to determine the effect of chemotherapy on immunological reactivity of tuberculosis (TB) patients to a wide variety of $M$. tuberculosis and non- $M$. tuberculosis antigens. Materials and Methods: The M. tuberculosis antigens were complex antigens (whole cells, sonicate, culture filtrate and cell wall) and single cytosolic, secreted and culture filtrate protein (CFP) antigens (a total of 19 purified protein antigens prepared by either biochemical fractionation or recombinant DNA technology). The non-M. tuberculosis antigens were Mycobacterium leprae proteins $(10,28,36$ and $65 \mathrm{kD})$, Escherichia coli heat shock protein (hsp) 65, Mycobacterium bovis hsp65 and maltose binding protein. Peripheral blood mononuclear cells (PBMC) obtained from active TB patients $(n=21)$ with moderate to far advanced disease, TB patients treated with anti-TB drugs for 3 months $(n=37)$, long-term healthy contacts of TB patients $(n=15)$ and healthy subjects from the general population $(\mathrm{n}=15)$ were tested for reactivity in antigen-induced proliferation and IFN- $\gamma$ assays. Antigen-induced T cell lines from the donor groups were established by stimulating PBMC with specific antigens. Overlapping synthetic peptides of MPT59, MPT64, ESAT6 and CFP-10 were used to map the T cell epitopes. Results: PBMC from active pretreated TB patients with moderate to far advanced disease showed low antigen-induced proliferation and IFN- $\gamma$ responses to the complex single antigens. Both of these responses were significantly elevated to some of the antigens after treating TB patients with anti-TB drugs for 3 months. Testing of PBMC and T cell lines with single antigens showed that the secreted antigens ESAT-6, MPT59, MPT64, MPB70 and CFP10 induced strong proliferation and IFN- $\gamma$ secretion. Several T cell epitopes of MPT59, MPT64, ESAT-6 and CFP10 were identified by testing the T cell lines with synthetic peptides. HLA typing analysis of PBMC demonstrated that these antigens were recognized by $T$ cell from individuals expressing a large spectrum of HLA-DR molecules. Conclusion: The results suggest that patients with active TB may have immunodeviation/immunosuppression to different types of $M$. tuberculosis antigens and effective anti-TB treatment restores the protective immunological response. Thus, anti-TB treatment could be beneficial to the patients in two ways: killing of tubercle bacilli through the direct effect of anti-TB drugs and restoration of immunological competence to further resist the spread of infection from the hidden sites in the body. In addition, the major $T$ cell antigens and epitopes identified in this study will be useful in developing new protective vaccines against $\mathrm{TB}$.

A.S. Mustafa (supervisor)

R. Al-Attiyah (co-supervisor)

Supported by Kuwait University's Research Administration grant MI114 and the College of Graduate Studies, Kuwait University, Kuwait.

\section{Polymerase Chain Reaction in the Diagnosis of Invasive Candidiasis}

\author{
A.I. Al-Rifaiy \\ Department of Microbiology, Faculty of Medicine, \\ Kuwait University, Kuwait
}

Objective: To establish a new format of seminested polymerase chain reaction (PCR) for detection and specific identification of four commonly encountered Candida species in clinical specimens. Materials and Methods: The seminested PCR was established by using universal fungal primers in the first round of amplification and species-specific primers of four Candida species, i.e. C. albicans, $C$. tropicalis, C. glabrata and C. parapsilosis, in the second round of amplification. The universal outer primers labeled with digoxigenin at the $5^{\prime}$-end amplified the 3 '-end of 5.8S ribosomal DNA (rDNA) and the 5 -end of $28 \mathrm{~S}$ rDNA, including the intervening internally transcribed spacer 2 (ITS2), generating 350- to 410-bp fragments. The species-specific primers labeled with biotin at the $5^{\prime}$-end were complementary to unique sequences within the ITS2 region of each test species. The products of seminested PCR were detected by agarose gel electrophoresis and ELISA. Blood samples of three groups of intravenously infected (normal, cyclophosphamide-treated, and cyclophosphamide and fluconazole-treated) mice, 40 in each group, and colonized mice $(\mathrm{n}=18)$ with $C$. albicans, and sera from blood culture-proven candidemia patients $(\mathrm{n}=30)$, superficially colonized $(n=6)$ patients, and healthy subjects $(n=10)$ were used for the evaluation of diagnostic efficacy of the seminested PCR. Results: The 
sensitivity of Candida detection by seminested PCR in spiked serum specimens was close to 1 organism $/ \mathrm{ml}$. Evaluation of seminested PCR for specific identification of Candida species with 50 clinical Candida isolates showed $99 \%$ concordant results with Vitek and/or ID32C yeast identification systems. The seminested PCR when applied for specific detection of $C$. albicans yielded positive results in $51(43 \%)$ mice as compared to $19(16 \%)$ by blood culture. The highest number of positive blood culture and seminested PCR results were obtained in cyclophosphamide-treated mice ( 8 and 20), followed by cyclophosphamide- and fluconazole-treated mice ( 6 and 15 ), and normal (5 and 16) mice, respectively. Among the gastrointestinally colonized mice fed on C. albicans and tetracycline dietary supplement, $C$. albicans DNA was detected in blood samples of 5 $(27.8 \%)$ of them. None of the control mice provided with distilled water or tetracycline yielded positive PCR results. The seminested PCR results were consistently negative in sera from healthy individu- als and colonized patients. In culture-proven candidemia patients, the seminested PCR results were in full agreement with blood culture results, both with respect to positivity and species specificity. The Candida species detected by seminested PCR included C. albicans $(\mathrm{n}=14), C$. parapsilosis $(\mathrm{n}=8)$ and C. glabrata $(\mathrm{n}=8)$. Conclusion: A highly sensitive and species-specific seminested PCR for the detection of four commonly encountered Candida species has been established and evaluated in this study. The established PCR assay will have a value in early and specific diagnosis of candidemia and invasive candidiasis.

\section{Z.U. Kahn (supervisor)}

A.S. Mustafa and S. Ahmad (co-supervisors)

The work was supported by the College of Graduate Studies and Research Administration (grant MI118), Kuwait University. 